This is an electronic reprint of the original article. This reprint may differ from the original in pagination and typographic detail.

Author(s): Räbinä, Jukka; Mönkölä, Sanna; Rossi, Tuomo

Title: $\quad$ High-quality discretizations for microwave simulations

Year: $\quad 2016$

Version:

Please cite the original version:

Räbinä, J., Mönkölä, S., \& Rossi, T. (2016). High-quality discretizations for microwave simulations. In Proceedings of 2016 URSI International Symposium on Electromagnetic Theory (EMTS), Espoo, Finland, 14-18 Aug 2016 (pp. 129-132). IEEE. https://doi.org/10.1109/URSI-EMTS.2016.7571332

All material supplied via JYX is protected by copyright and other intellectual property rights, and duplication or sale of all or part of any of the repository collections is not permitted, except that material may be duplicated by you for your research use or educational purposes in electronic or print form. You must obtain permission for any other use. Electronic or print copies may not be offered, whether for sale or otherwise to anyone who is not an authorised user. 


\title{
High-Quality Discretizations for Microwave Simulations
}

\author{
Jukka Räbinä, Sanna Mönkölä*, and Tuomo Rossi \\ University of Jyvaskyla, Department of Mathematical Information Technology, \\ P.O. Box 35 (Agora), FI-40014 University of Jyväskylä, Finland \\ * e-mail: sanna.monkola@jyu.fi
}

\begin{abstract}
We apply high-quality discretizations to simulate electromagnetic microwaves. Instead of the vector field presentations, we focus on differential forms and discretize the model in the spatial domain using the discrete exterior calculus. At the discrete level, both the Hodge operators and the time discretization are optimized for time-harmonic simulations. Non-uniform spatial and temporal discretization are applied in problems in which the wavelength is highly-variable and geometry contains sub-wavelength structures.
\end{abstract}

\section{INTRODUCTION}

Microwaves are high-frequency electromagnetic waves that can propagate in any spatial direction. Thus, it is important to maintain uniform physical properties in all directions, which is not the case when the cubic grid is applied in the finitedifference time-domain (FDTD) method, originally proposed by Yee [1]. The quality of the discrete model, based on the structure of the computational grid, has a significant impact on the efficiency of the method. We use non-uniform grids and associate the degrees of freedom of the electric and magnetic fields with primal and dual grids, respectively. The physical characterization of the discretization is presented by the discrete Hodge operators defining the connection between the primal and dual forms [2]. The discretization is independent of the coordinate systems, and by the orthogonality (with respect to metric) of the primal and dual mesh elements, we ensure the diagonal construction of Hodge operators providing significant savings in computing time [3].

\section{Electromagnetics in the Time Domain}

The work needed to move a particle with electric charge $q$, along an oriented curve $\mathcal{C}$, in an electric field $\mathbf{E}=$ $\left(E_{1}, E_{2}, E_{3}\right)^{T}$ is

$$
W=-q \int_{\mathcal{C}} \mathbf{E} \cdot d l=-q \int_{\mathcal{C}} E
$$

where $d l$ is the differential unit tangent vector of the curve, $d x_{i}, i=1,2,3$, are differentials and $E=E_{1} d x_{1}+E_{2} d x_{2}+$ $E_{3} d x_{3}$ is a differential 1-form. Respectively, we can consider a magnetic charge and obtain a differential 1-form $H=$ $H_{1} d x_{1}+H_{2} d x_{2}+H_{3} d x_{3}$ (instead of the magnetic field $\mathbf{H}$ ).

The current flowing through a surface $\mathcal{S}$

$$
I=\int_{\mathcal{S}} \mathbf{J} \cdot d \mathcal{A}=\int_{\mathcal{S}} J
$$

where $d \mathcal{A}=\mathbf{n} \cdot d \mathcal{S}$ is the differential vector element of surface area $\mathcal{A}$ normal to surface $\mathcal{S}, \wedge$ is the exterior product (wedge product), and electric current density $J=J_{1} d x_{2} \wedge$ $d x_{3}+J_{2} d x_{3} \wedge d x_{1}+J_{3} d x_{1} \wedge d x_{2}$ is a differential 2-form. Respectively, we can define the magnetic current density $J^{*}$. The electric charge in volume $\mathcal{V}$ is

$$
q=\int_{\mathcal{V}} \varrho d \mathcal{V}=\int_{\mathcal{V}} Q
$$

where $\varrho$ is the electric charge density and $Q=\varrho d x_{1} \wedge d x_{2} \wedge$ $d x_{3}$ is a differential 3 -form presenting the electric charge. Respectively, $Q^{*}=\varrho^{*} d x_{1} \wedge d x_{2} \wedge d x_{3}$ the magnetic charge.

The generalized Stokes theorem, applied to a differential form $A$ over the boundary of an oriented manifold $\Omega$, is

$$
\oint_{\partial \Omega} A=\int_{\Omega} d A,
$$

where $d$ is the exterior derivative. The Hodge star $\star$, defining the coordinate system and metric properties, maps a differential $k$-form to a differential $n-k$ form ( $n=3$ is the spatial dimension). The constitutive relations are written as $D=\star \varepsilon E$ and $B=\star \mu H$, where $D$ and $B$ are differential 2-forms and $\varepsilon$ is the electric permittivity and $\mu$ is the magnetic permeability. The electromagnetic waves are presented by

$$
\begin{aligned}
& \star \varepsilon \frac{\partial E}{\partial t}-d H=-J, \\
& \star \mu \frac{\partial H}{\partial t}+d E=-J^{*}, \\
& d D=Q, \quad d B=Q^{*},
\end{aligned}
$$

where the exterior derivative $d$ on 1 -forms corresponds to the curl operator on vector fields whereas on 2 -forms it corresponds to the div operator on vector fields. By taking the exterior derivative of Equations (5) and (6), we get the continuity of the charges,

$$
\frac{\partial Q}{\partial t}=-d J, \quad \frac{\partial Q^{*}}{\partial t}=-d J^{*},
$$

and the information given by Equations (7) can be covered by the initial conditions. 


\section{DiscretizATION}

The computational domain $\Omega$ is discretized by a mesh, in which we have vertices, edges, surfaces, and volumes. In particular, we are interested in the edge elements $\mathcal{E}_{j}$, $j=1, \ldots, N_{\mathcal{E}}$ and face elements $\mathcal{F}_{i}, i=1, \ldots, N_{\mathcal{F}}$, where $N_{\mathcal{E}}$ and $N_{\mathcal{F}}$ are the number of the edge and face elements, respectively. We also construct a dual mesh with edge elements $\mathcal{E}_{i}^{*}$, which are duals to the primal face elements, and face elements $\mathcal{F}_{j}^{*}$, which are duals to the primal edge elements.

\section{A. Discrete Exterior Calculus}

The spatial discretization is applied using the discrete exterior calculus (DEC) [4]. The discrete 1-form $E$ and the discrete 2-forms $\mathrm{B}$ and $\mathrm{J}^{*}$ are presented as column vectors of components

$$
\mathrm{E}_{j}=\int_{\mathcal{E}_{j}} E, \quad \mathrm{~B}_{i}=\int_{\mathcal{F}_{i}} B, \quad \mathrm{~J}_{i}^{*}=\int_{\mathcal{F}_{i}} J^{*} .
$$

The discrete exterior derivative $d$ (boundary operator) is a $N_{\mathcal{F}} \times N_{\mathcal{E}}$ matrix presenting the incidence number of each $\mathcal{E}_{j}$ to each face $\mathcal{F}_{i}$. The incidence number is 0 if $\mathcal{E}_{j}$ is not a boundary of $\mathcal{F}_{i}$. If $\mathcal{E}_{j}$ is a boundary of $\mathcal{F}_{i}$, the value at the $i$ th row and the $j$ th column of the incidence matrix is \pm 1 , depending on the relative orientation between the face and the edge element. The spatially discretized formulation of Equation (6) is

$$
\frac{\partial}{\partial t} \mathrm{~B}+\mathrm{dE}=-\mathrm{J}^{*}
$$

On the dual mesh, we have discrete dual forms. The discrete dual 1-form $\mathrm{H}$ and the discrete dual 2-forms $\mathrm{D}$ and $\mathrm{J}$ are presented as column vectors of components

$$
\mathrm{H}_{i}=\int_{\mathcal{E}_{i}^{*}} H, \quad \mathrm{D}_{j}=\int_{\mathcal{F}_{j}^{*}} D, \quad \mathrm{~J}_{j}=\int_{\mathcal{F}_{j}^{*}} J,
$$

The elements of the dual mesh have a similar orientation to the corresponding primal elements if the incidence number of each $\mathcal{E}_{j}$ to each face $\mathcal{F}_{i}$ equals to the incidence number of each $\mathcal{E}_{i}^{*}$ to each face $\mathcal{F}_{j}^{*}$. Then, the dual discrete exterior derivative is $\mathrm{d}^{T}$ and the spatially discretized formulation of Equation (5) is presented as

$$
\frac{\partial}{\partial t} \mathrm{D}-\mathbf{d}^{T} \mathrm{H}=-\mathrm{J}
$$

The discrete Hodge stars are needed to present the constitutive relations $\mathrm{D}=\star \varepsilon \mathrm{E}$ and $\mathrm{B}=\star \mu \mathrm{H}$. The $\varepsilon$-related discrete Hodge $\star \varepsilon$ is a $N_{\mathcal{E}} \times N_{\mathcal{E}}$ diagonal matrix, presenting the linear map from the discrete primal 1-form $E$ to the discrete dual 2-form $D$. The diagonal terms are

$$
\star \varepsilon_{j j}=\varepsilon \frac{\left|\mathcal{F}_{j}^{*}\right|}{\left|\mathcal{E}_{j}\right|} \kappa_{j},
$$

where the curvature correction $\kappa_{j}$ is set to compensate for the systematic wave speed error of harmonic wave. As derived in [3],

$$
\kappa_{j}=\left(\frac{1-\frac{\kappa_{\mathcal{F}}}{5}+\frac{\kappa_{\mathcal{F}}^{2}}{56}}{1-\frac{\kappa_{\mathcal{F}}}{10}-\frac{\kappa_{\mathcal{E}}}{120}+\frac{\kappa_{\mathcal{F}}^{2}}{280}+\frac{\kappa_{\mathcal{F}} \kappa_{\mathcal{E}}}{1680}+\frac{\kappa_{\mathcal{E}}^{2}}{22400}}\right),
$$

where $\kappa_{\mathcal{E}}=\omega^{2} \epsilon \mu\left|\mathcal{E}_{j}\right|^{2}$ and $\kappa_{\mathcal{F}}=\omega^{2} \epsilon \mu\left(2 r^{2}+R^{2}\right) / 3$, and $r$ and $R$ are the inner and outer radii of dual face $\mathcal{F}_{j}^{*}$. The $\mu$-related discrete Hodge operator $\star \mu$ is a $N_{\mathcal{F}} \times N_{\mathcal{F}}$ diagonal matrix mapping the discrete dual 1-form $\mathrm{H}$ to the discrete primal 2-form B presented as an $N_{\mathcal{F}} \times N_{\mathcal{F}}$ diagonal matrix

$$
\star \mu_{i i}=\mu \frac{\left|\mathcal{F}_{i}\right|}{\left|\mathcal{E}_{i}^{*}\right|} \kappa_{i}^{*},
$$

where the curvature correction $\kappa_{i}^{*}$ is derived in a similar manner with respect to the elements $\mathcal{F}_{i}$ and $\mathcal{E}_{i}^{*}$.

Applying DEC on a regular grid without curvature correction results in FDTD discretization as a special case. The curvature correction and high-quality meshing can increase the method efficiency by orders of magnitude [3].

\section{B. Time Discretization}

The time evolution begins with the initial conditions $\mathrm{E}^{0}=$ $\mathrm{E}\left(-\frac{\Delta t}{2}\right)$ and $\mathrm{H}^{0}=\mathrm{H}(0)$, after which it proceeds, as a staggered procedure, by computing $\mathrm{E}^{k}=\mathrm{E}\left(k \Delta t-\frac{\Delta t}{2}\right)$ and $\mathrm{H}^{k}=\mathrm{H}(k \Delta t)$, where $k=0,1,2, \ldots$ and $\Delta t$ is the length of the time step. Since we are considering time-harmonic simulations, we optimize the time discretization for a single wave frequency. That is, instead of the conventional leapfrog time-discretization, we apply the formulas

$$
\begin{aligned}
\mathrm{E}\left(t^{k}\right) & =\frac{\mathrm{E}^{k}+\mathrm{E}^{k+1}}{2 \cos \frac{\omega \Delta t}{2 s}}, \quad \mathrm{H}\left(t^{k}+\frac{\Delta t}{2}\right)=\frac{\mathrm{H}^{k}+\mathrm{H}^{k+1}}{2 \cos \frac{\omega \Delta t}{2 s}}, \\
\frac{\partial \mathrm{E}}{\partial t}\left(t^{k}\right) & =\frac{\mathrm{E}^{k+1}-\mathrm{E}^{k}}{\frac{2}{\omega} \sin \frac{\omega \Delta t}{2 s}}, \frac{\partial \mathrm{H}}{\partial t}\left(t^{k}+\frac{\Delta t}{2}\right)=\frac{\mathrm{H}^{k+1}-\mathrm{H}^{k}}{\frac{2}{\omega} \sin \frac{\omega \Delta t}{2 s}},
\end{aligned}
$$

where $s$ is the time step size factor [3]. For uniform grids, we can choose $s=1$ and apply the CFL condition to get the upper bound for $\Delta t$.

Since we use non-uniform space discretization, and the time step size needs to be adjusted to the wavelength, we allow smaller time steps for the finer parts of the grid. The local time steps are obtained from the localized CFL-type condition, which take only a small neighborhood of elements into account [3]. The time step is adjusted by the integer-valued factors $s_{E_{j}}$ and $s_{H_{i}}$, such that, for updating the values of $\mathrm{E}_{j}^{k}$, we use $s=s_{E_{j}}$ and for updating the values of $\mathrm{H}_{i}^{k}$, we use $s=s_{H_{i}}$. To guarantee the energy conservation, we synchronize the time stepping by setting $s_{E_{j}}, s_{H_{i}} \in\{1,3,9,27, \ldots\}$. Using the time stepping approach, we obtain an explicit and causal simulation method, which can be efficiently applied to solve time-harmonic problems [3].

\section{NUMERICAL EXPERIMENTS}

The DEC discretization can be applied for arbitrary polyhedral meshing with orthogonal duality. The dual mesh is obtained as a Voronoi diagram generated for a given vertex set and given metric induced by the material. The primal mesh is determined by the topology. The numerical experiments of this section exploit the body-centered cubic (BCC) structure [5], [6] defining the primal grid with congruent tetrahedra and the dual grid with truncated octahedra [7]. A uniform grid is rarely optimal discretization for numerical simulations. Thus, 
we apply structured grid refining, where vertex structures are applied in different scales. In such a manner, one can efficiently obtain non-uniform grids, where element properties are guaranteed. We apply grid refinement due to the highly variable material parameters and for the discretization of subwavelength structures.

\section{A. Highly variable material parameters}

We consider a microwave oven, of which the dimensions are $28.0 \times 28.0 \times 21.0$ centimeters representing depth $x$, width $y$, and height $z$, respectively. Placed in the middle of the wall facing in the positive $y$-direction, a square-shaped magnetron with an edge length of $12.2 \mathrm{~cm}$ produces a harmonic wave of frequency $f=2.45 \mathrm{GHz}$, implying that the wavelength is $\lambda=12.2 \mathrm{~cm}$. Inside the microwave oven, centered at height of $3 \mathrm{~cm}$ above the bottom of the computational domain, is a bowl, having the shape of a conical frustum. The bowl is filled with liquid with a depth of $6 \mathrm{~cm}$; the bottom radius is $3 \mathrm{~cm}$ and the top radius is $6 \mathrm{~cm}$. We approximate the liquid by permittivity $\varepsilon=70 \varepsilon_{0}$, where $\varepsilon_{0}$ is the air (or vacuum) permittivity. The absorption term of the liquid is determined by the right-hand side source term $J=0.58 \omega \varepsilon_{0} E$.

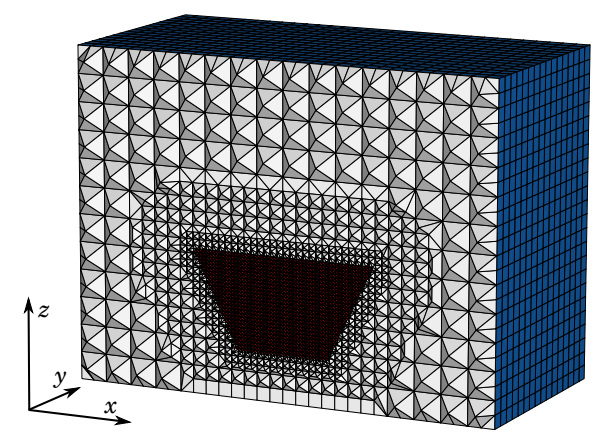

Fig. 1. Cross section of the applied mesh. The interior is filled with structured BCC grid, and the structured grid refinement strategy is applied near the bowl.

The structured grid refining was applied involving at least 11,000 unknowns per $\lambda^{3}$ and a total of 2,005,708 unknowns in the domain (see Fig. 1). The perfectly reflecting walls are defined by the Dirichlet boundary condition. The magnetron is modeled by the absorbing boundary condition with source terms $E_{0}=(\cos \omega t, 0, \sin \omega t)^{T}, H_{0}=(\sin \omega t, 0,-\cos \omega t)^{T}$, where $\omega=2 \pi f$ is the angular frequency.

The numerical experiment was initialized by $E^{0}=0$ and $\mathrm{H}^{0}=0$, and was continued until time $t=501 T$ was reached, where $T=1 / f$ is the wave period. During the first period, the source terms were increased from zero to full terms by the transitions suggested in [8]. For the last 500 iterations, the full source terms were applied. The time step size factor $s$ varied between 1 and 27 and a total of 205,557,312 value updates were executed per iteration. The simulation was carried out in around 4 minutes on 32 Intel Xeon cores at $2.67 \mathrm{GHz}$. The results, presenting the cross sections of electric and magnetic fields, are illustrated in Fig. 2.

In comparison, an FDTD simulation with ten elements per wavelength means 6000 unknowns per $\lambda^{3}$. The liquid
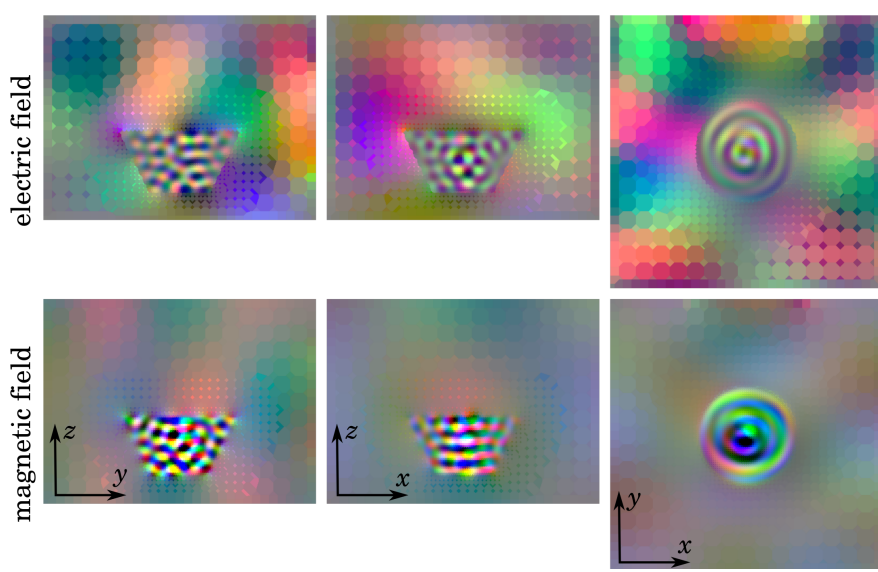

Fig. 2. Cross sections of electric and magnetic fields are illustrated on three different planes. The color components red, green, and blue represent the values of $x, y$, and $z$ components of the resulting vector. Grey denotes the zero field.

wavelength is $1.46 \mathrm{~cm}$ designating 1940 unknowns per $\mathrm{cm}^{3}$. That makes about 31.9 million unknowns in the whole domain. Such a grid has 83.7 elements per vacuum wavelength, and therefore the CFL condition requires 145 time steps per period. In total, the FDTD simulation would take 4.62 billion value updates per period, which is more than 20 times the number of value updates in our numerical simulation.

\section{B. Sub-wavelength structure}

In $x$-direction, the domain is a three-layer structure with thickness 1 truncated by the absorbing boundary condition. The back and front layers are discretized by $y$ - and $z$-periodic mesh, as illustrated in Fig. 3, and the source term for a circularly polarized plane wave of wavelength $\lambda=1$ is set at the back wall. The middle layer is a plane of a periodic sub-wavelength grille structure with circular holes surrounded by perfectly conducting material $(E=0)$. For the numerical tests, we apply six different grilles, where the periodic pattern is repeated $1,2,4,8,16$, or 40 times per unit length.

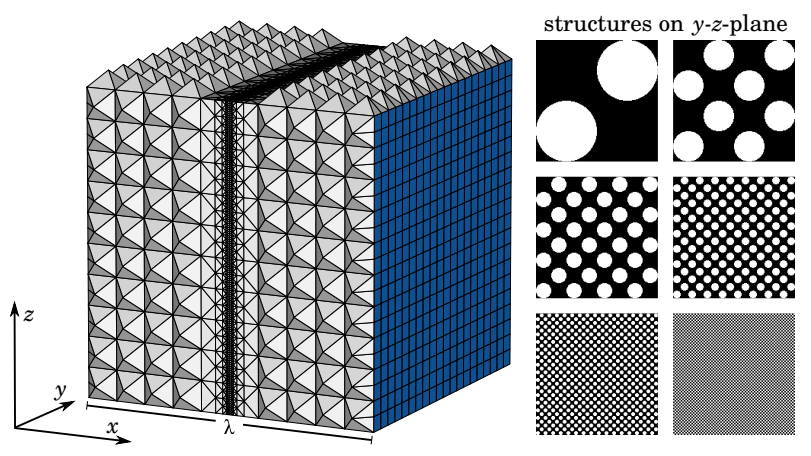

Fig. 3. The $y$-z-periodic mesh for discretization level 10 and the subwavelength grilles are illustrated on the left and right, respectively.

We apply four discretization levels, which are called 5, 10, 20 and 40. Each discretization has a base grid of the BCC structure, where the maximum edge lengths are $\frac{1}{5}, \frac{1}{10}, \frac{1}{20}$, and $\frac{1}{40}$, respectively. Structured grid refinement is applied near the plane $x=0$ by inserting vertices of the regular structure 

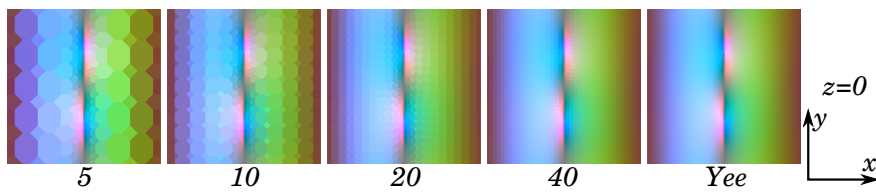

Fig. 4. Cross sections of electric fields for different discretization levels. Red green, and blue represent $x$-, $y$-, and $z$-components of the field, respectively. of step size $h=\frac{2^{n}}{160}, n=0,1, \ldots$ Each regular group of vertices is limited by $x \in[-h, h]$. The reference solution is computed using an ultra-fine Cartesian discretization, where the edge lengths throughout the domain are $\frac{1}{160}$. The numbers of unknowns are $899,150,927,600,1,174,400,3,225,600$, and $24,652,800$ for the different discretization levels, respectively.

The simulations were initialized by $\mathrm{E}^{0}=0$ and $\mathrm{H}^{0}=0$ and processed by 10 periods with increasing source terms and by 10 periods with full source terms. The solutions for each discretization level and for the largest grille size are illustrated in Fig. 4. The energy penetration is computed for each grille and each discretization level and the results are illustrated in Fig. 5. The larger the holes are, the more energy penetrates, and for the largest hole size the penetration is approximately $50 \%$. This is surprising, as holes cover only $\frac{\pi}{8} \approx 39 \%$ of the whole surface.

The solutions for each discretization level are close to each other, but upon closer examination, there is up to $25 \%$ relative difference between the results. The conclusion is that the refinement should cover a wider volume. We increase the depth of the refinement such that each regular group of nodes is limited by $x \in[-k h, k h]$, where $k=1,2,3,4$ is called the refinement thickness. Figure 6 shows that wider refinement improves the accuracy. With the largest refinement width, one has less than $1 \%$ error. The number of unknowns are $1,174,400,1,466,400,1,854,400$, and $2,146,400$ for these cases, respectively, which are less than one tenth compared to the reference discretization.

Discretization level: $\diamond 5 * 10 \bullet 20 \bullet 40--$ reference
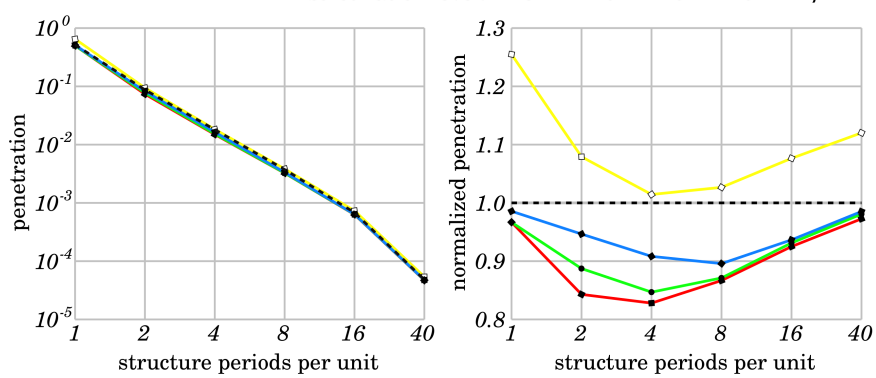

Fig. 5. Penetration of the wave energy by grille size. The normalized penetration illustrates the discretization error compared to the reference solution.

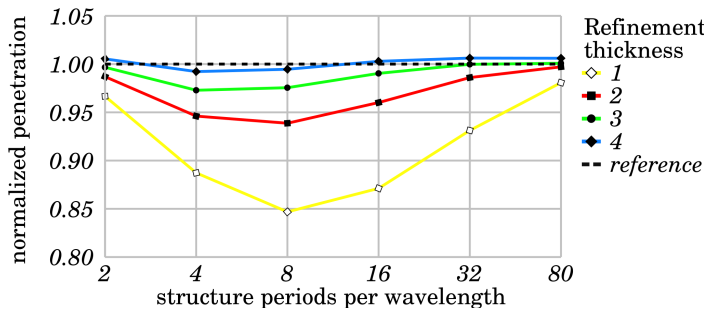

Fig. 6. Discretization error by refinement thickness.

\section{Combined problem}

We repeat the microwave simulation by applying a grille with circular holes with diameter $0.22 \mathrm{~cm}$ on the wall facing in positive $x$-direction. The elements size at the grille is 0.11 $\mathrm{cm}$ and the discretization is illustrated in Fig. 7. The number of unknowns is $3,729,426$ and the time step size factor $s$ vary between 1 and 27 . The total number of value updates per period is $751,607,208$. The simulation through $501 T$ took about 14 minutes on 32 Intel Xeon cores at $2.67 \mathrm{GHz}$. As a result, $0.063 \%$ of the power produced by the magnetron is penetrated through the grille. The rest is absorbed as heat in the liquid. The energy loss is so small that the grille does not have a remarkable effect on the progress of electromagnetic energy inside the liquid, as illustrated in Fig. 7.
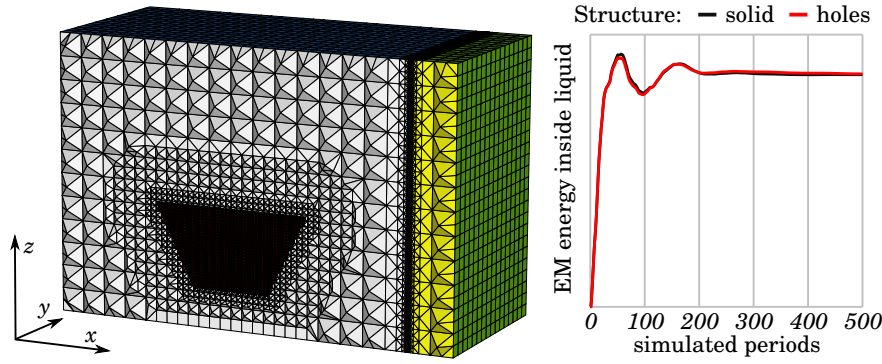

Fig. 7. Cross section of the mesh with the refined layer on one side. The progress of electromagnetic energy inside the liquid area is illustrated on the right.

\section{CONCLUSIONS}

We applied polyhedral structures and grid-refining methods with DEC. The grid refinement is useful in modeling tasks with highly variable material parameters and sub-wavelength structures. Further research should consider how the error estimation methods can be applied to find the optimal refinement.

\section{ACKNOWLEDGMENT}

This research is supported by the Academy of Finland contract 259925.

\section{REFERENCES}

[1] K. S. Yee, "Numerical solution of initial boundary value problems involving Maxwell's equations in isotropic media," IEEE Transaction on antennas and propagation, vol. 14, no. 3, pp. 302-307, 1966.

[2] A. Bossavit and L. Kettunen, "Yee-like schemes on staggered cellular grids: A synthesis between FIT and FEM approaches," IEEE Transactions on Magnetics, vol. 36, no. 4, pp. 861-867, 2000.

[3] J. Rabina, S. Monkola, and T. Rossi, "Efficient time integration of Maxwell's equations with generalized finite differences," SIAM Journal on Scientific Computing, vol. 37, no. 6, pp. B834-B854, 2015.

[4] M. Desbrun, A. N. Hirani, M. Leok, and J. E. Marsden, "Discrete exterior calculus," 2005, preprint, arXiv:math/0508341v2 [math.DG]

[5] D. M. Y. Sommerville, "Space-filling tetrahedra in Euclidean space," Proceedings of the Edinburgh Mathematical Society, vol. 41, pp. 49-57, 21922.

[6] J. Conway and N. J. A. Sloane, Sphere Packings, Lattices and Groups, 3rd ed., ser. A series of comprehensive studies in mathematics. Springer, 1999.

[7] W. Thomson, "On the division of space with minimum partitional area," Acta Mathematica, vol. 11, no. 1-4, pp. 121-134, 1887.

[8] G. Mur, "The finite-element modeling of three-dimensional electromagnetic fields using edge and nodal elements," IEEE Transactions on Antennas and Propagation, vol. 41, no. 7, pp. 948-953, 1993. 